\title{
Cerebral systemic lupus erythematosus presenting with catatonia
}

\author{
J.G. Lanham ${ }^{1 *}$, M.M. Brown ${ }^{2}$ and G.R.V. Hughes ${ }^{1}$ \\ ${ }^{1}$ Rheumatology Unit and ${ }^{2}$ Neurology Unit, Royal Postgraduate Medical School, Hammersmith Hospital, Du Cane \\ Road, London W12 0HS, UK.
}

\begin{abstract}
Summary: A 13 year old girl with catatonia resulting from cerebral lupus is described. She had concurrent minor epileptic status, but abolition of her seizure activity failed to influence her catatonic state. She recovered after treatment with corticosteroids and immunosuppressive agents. Cerebral lupus should be considered in the differential diagnosis in patients presenting with catatonia.
\end{abstract}

\section{Introduction}

Catatonia is a clinical syndrome characterized by psychosocial withdrawal, negativism, mutism and posturing, together with signs of rigidity and waxy flexibility. There may also be periods of excitement or bizarre repetitious behaviour. Catatonia is frequently assumed to be diagnostic of schizophrenia, although it is known to occur rarely in a number of metabolic and structural diseases of the brain (Gellenberg, 1976). We report a case of catatonia resulting from cerebral involvement by systemic lupus erythematosus (SLE), in which investigation confirmed the organic nature of the disease.

\section{Case report}

A 13 year old Cypriot girl presented with a $24 \mathrm{~h}$ history of psychological withdrawal. For 2 days prior to this she had been excitable, exhibiting outbursts of violent behaviour. Eighteen months previously she had developed a facial rash, vomiting and ankle swelling and had been diagnosed elsewhere as suffering from systemic lupus erythematosus. These symptoms remitted after a course of steroids but recurred three months

J.G. Lanham, M.B., B.Ch., M.R.C.P.; M.M. Brown, B.A., M.B., B.S., M.R.C.P.; G.R.V. Hughes, M.D., F.R.C.P.

*Correspondence \& present address: Department of Rheumatology, St Bartholomew's Hospital, London. EC1A 7BE, UK.

Accepted: 19 April 1984 before the current presentation. She had been noted at that time to have Raynaud's phenomenon, cutaneous photosensitivity, scalp alopecia, vasculitic lesions on the extremeties, hepatosplenomegaly and proteinuria. Steroid therapy again induced a clinical remission and she remained well on a maintenance dose of $30 \mathrm{mg}$ prednisolone per day without behavioural or mood disturbance until 3 days before the current admission. There was no family history of psychiatric illness, epilepsy or connective tissue disease, although her mother suffered from immune thrombocytopenic purpura.

On examination the patient was conscious, but withdrawn and vacant. She was completely mute and did not obey commands. Periodically she would move her eyes conjugately as if inspecting her surroundings, but would only occasionally and briefly fixate on an object or her attendants. She made no other spontaneous movement, but would stand if lifted out of bed and would take a few steps if led. The tone in all limbs was variably increased and she demonstrated waxy flexibility, maintaining indefinitely any posture into which her limbs were placed. In the optic fundi bilateral soft exudates were observed and she had bilateral extensor plantar responses. The remainder of the neurological examination was normal. She was afebrile and no abnormalities were found in the cardiovascular, respiratory or abdominal systems. The cutaneous features of systemic lupus erythematosus noted previously had regressed apart from residual scalp alopecia.

(C) The Fellowship of Postgraduate Medicine, 1985 
Investigations revealed a microcytic anaemia with a haemoglobin of $8.9 \mathrm{~g} / \mathrm{dl}$; white blood count of $9.6 \times 10^{9} / 1$ and platelet count of $228 \times 10^{9} / 1$. The erythrocyte sedimentation rate was raised at $146 \mathrm{~mm}$ in the first hour. Plasma urea, electrolytes, glucose, liver function tests, urate, calcium, phosphate, creatinine clearance, urinary protein excretion, chest X-ray and electrocardiogram were all normal. DNA binding was significantly elevated at $97 \%$ (normal range below $30 \%$ ) and antibodies to RNP and Sm antigens were detected. $\mathrm{C} 3$ and $\mathrm{C} 4$ components of complement were substantially reduced and hypergammaglobulinemia was present. Serological testing for syphilis was negative.

Examination of the cerebrospinal fluid, including virological studies, was normal. A cranial computerized tomographic (CT) scan with contrast enhancement showed widening of the cortical sulci with small ill-defined non-enhancing low attenuation lesions in the parietal and temporal regions of both hemispheres. An electroencephalogram (EEG) showed she was having very frequent subclinical epileptic seizures characterized by bursts of high voltage slow spike and wave activity $(1.5-2 \mathrm{~Hz})$, maximal and focal in the left fronto-parietal region; this was abolished by an intravenous injection of diazepam.

In view of the EEG findings, treatment was commenced with oral clonazepam, in addition to prednisolone $40 \mathrm{mg}$ daily. This resulted in sustained abolition of all seizure activity from the EEG, but there was no change in her clinical signs. The dose of prednisolone was therefore increased to $60 \mathrm{mg} / \mathrm{d}$ and she received a single intravenous bolus of $400 \mathrm{mg}$ cyclophosphamide. Within 3 days the catatonic features diminished and she began to respond to simple commands. Treatment with azathioprine was then introduced and she made a gradual recovery. Six weeks after presentation she was almost completely normal and 6 months later she was well enough to obtain excellent results in the end of year school examinations.

\section{References}

BENNETT, R. HUGHES, G.R.V., BYWATERS, E.G.L. \& HOLT, P.J.L. (1972). Neuropsychiatric problems in systemic lupus erythematosus. British Medical Journal, 4, 342.

CARETTE, S., UROWITZ, M.B., GROSMAN, H. \& ST LOUIS, E.L. (1982). Cranial computerised tomography in systemic lupus erythematosus. Journal of Rheumatology, 9, 855.

GELLENBERG, A.J. (1976). The catatonic syndrome. Lancet, i, 1339.

FEINGLASS, E.J., ARNETT, F.C., DORSCH, C.A., ZIZIC, M. \& STEVENS, M.B. (1976). Neuropsychiatric manifestations of

\section{Discussion}

This patient presented with a catatonic syndrome, but the clinical and serological features of her preceding illness were typical of SLE. The brain is commonly involved in SLE, and she had obvious organic central nervous system disease. The cranial CT scan appearances were compatible with cerebral lupus (Cavette et al., 1982) and seizure activity is a common manifestation of the condition. Although epilepsy may result in catatonia (Shah \& Kaplan, 1980) this did not appear to be the case in our patient, because successful treatment of the seizure activity had no impact on her clinical state. However, the occurrence of epilepsy in cerebral lupus is frequently associated with other neuropsychiatric manifestations (Feinglass et al., 1976) and it is therefore likely that the catatonic state and the seizure activity in our patient were independent manifestations of this condition. Psychiatric disorders are well recognized in SLE, and catatonia has previously been reported (Kronfol et al., 1977) although not in association with seizure activity or CT scan abnormalities. The successful response to immunosuppression and increased steroid dosage makes it extremely unlikely that the catatonia was a manifestation of a steroid psychosis.

This case history illustrates the importance of considering organic disease in patients presenting with catatonia. Cerebral lupus may occur without signs of disease activity in other organs, and psychotic beto haviour may precede the development of other: features of the condition by months or years (Bennett et al., 1972). Systemic lupus erythematosus should therefore be included in the differential diagnosis of patients presenting with the catatonic syndrome.

\section{Acknowledgements}

We thank Dr C. Pallis and Mrs Valerie Emery for helpful advice.

systemic lupus erythematosus. Diagnosis, clinical spectrum and relationship to other features of the disease. Medicine (Baltimore), 55, 323.

KRONFOL, Z., SCHLESSER, M. \& TSUANG M.T. (1977). Catatonia and systemic lupus erythematosus. Diseases of the Nervous System, 38, 729.

SHAH, P. \& KAPLAN, S.L. (1980). Catatonic syndrome in a child with epilepsy. American Journal of Psychiatry, 137, 738 . 